\title{
On the chromatic number of set systems
}

\author{
Alexandr Kostochka*, Dhruv Mubayi ${ }^{\dagger}$, Vojtĕch Rödl ${ }^{\ddagger}$ Prasad Tetali ${ }^{\S}$
}

April 20, 2005

\begin{abstract}
An $(r, l)$-system is an $r$-uniform hypergraph in which every set of $l$ vertices lies in at most one edge. Let $m_{k}(r, l)$ be the minimum number of edges in an $(r, l)$-system that is not $k$-colorable. Using probabilistic techniques, we prove that

$$
a_{r, l}\left(k^{r-1} \ln k\right)^{\frac{l}{l-1}} \leq m_{k}(r, l) \leq b_{r, l}\left(k^{r-1} \ln k\right)^{\frac{l}{l-1}}
$$

where $b_{r, l}$ is explicitly defined and $a_{r, l}$ is sufficiently small. We also give a different argument proving (for even $k$ )

$$
m_{k}(r, l) \geq a_{r, l}^{\prime} k^{\frac{(r-1) l}{l-1}}
$$

where $a_{r, l}^{\prime}=\frac{r-l+1}{r}\left(2^{r-1} r e\right)^{\frac{-l}{l-1}}$.

Our results complement earlier results of Erdős and Lovász [10] who mainly focused on the case $l=2, k$ fixed, and $r$ large.
\end{abstract}

\section{Introduction}

A hypergraph $H$ is $k$-colorable if its vertex set can be partitioned into $k$ color classes, such that no edge is monochromatic. The chromatic number $\chi(H)$ of $H$ is the minimum $k$ such

*Department of Mathematics, University of Illinois, Urbana, IL 61801 and Institute of Mathematics, Novosibirsk, 630090, Russia; research supported in part by grants 99-01-00581 and 00-01-00916 of the RFBR.

${ }^{\dagger}$ School of Mathematics, Georgia Institute of Technology, Atlanta, GA 30332-0160; research supported in in part by NSF Grant No. DMS-9970325.

${ }_{\ddagger}^{\ddagger}$ Department of Mathematics, Emory University, Atlanta, GA 30322; research supported in part by NSF Grant No. DMS-0071261.

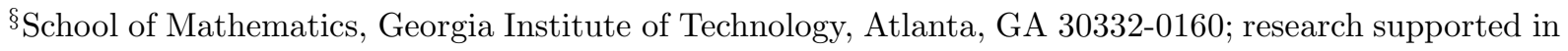
in part by NSF Grant No. DMS-9800351.

1991 Mathematics Subject Classification: O5B07, 05C35, 05C65, 05D05, 05D15

Keywords: Extremal set theory, uniform hypergraph, property $B$ 
that $H$ is $k$-colorable. A classical extremal problem is to determine the minimum number of edges in an $r$-uniform hypergraph ( $r$-graph for short) that is not $k$-colorable. This minimum has been denoted $m_{k}(r)$ (see $[2,4,6,8,9,12,14]$ for the results in the case $k=2$ and [1] for large $k$ ). If we restrict to the class of simple hypergraphs, i.e., those where every two distinct vertices lie in at most one edge, then the corresponding parameter is denoted by $m_{k}^{*}(r)$. This parameter was first studied by Erdős and Lovász [10]. They proved the bounds

$$
\frac{k^{2(r-2)}}{16 r(r-1)^{2}} \leq m_{k}^{*}(r) \leq 1600 r^{4} k^{2(r+1)},
$$

which imply that

$$
\lim _{r \rightarrow \infty} m_{k}^{*}(r)^{1 / r}=k^{2} .
$$

We consider a larger class of hypergraphs. A partial $(r, l)$-system (henceforth, $(r, l)$ system), is an $r$-uniform hypergraph in which every set of $l$ vertices lies in at most one edge. Let $m_{k}(r, l)$ be the minimum number of edges in an $(r, l)$-system that is not $k$-colorable; thus $m_{k}^{*}(r)=m_{k}(r, 2)$.

The works $[13,15,11]$ on Steiner systems with small independence number yield results for $(r, l)$-systems, and imply upper bounds on $m_{k}^{*}(r)$ which improve (1) for $k$ very large in comparison with $r$. In particular, Grable, Phelps and Rödl [11] for every $r$ and infinitely many $k$ constructed simple hypergraphs (in fact, Steiner systems) with chromatic number at least $k+1$ and at most $c 4^{r} r^{2} k^{2 r-2} \ln ^{2} k$ edges. Thus, for such $r$ and $k$,

$$
m_{k}^{*}(r) \leq c 4^{r} r^{2} k^{2 r-2} \ln ^{2} k .
$$

Our first result improves the upper bound in (1) in the range $k^{4}>0.01 r^{6}\left(\ln ^{2} e k\right)$. It has the advantage over (2) that it applies for every $l \geq 2$. We write $(r)_{l}$ for $r(r-1) \cdots(r-l+1)$.

Theorem 1 Let $r \geq 3, l \geq 2$. Then

$$
m_{k}(r, l) \leq 2 \frac{\left(c_{r, l}\right)^{l}}{(r)_{l}}\left(k^{r-1} \ln e k\right)^{\frac{l}{l-1}},
$$

where $c_{r, l}=\left(2 r^{3 l}\right)^{\frac{1}{l-1}}$.

We also improve the lower bound in (1) for $r \geq 3$ and large $k$.

Theorem 2 Let $r \geq 3$. If $k$ is even, then

$$
m_{k}(r, l) \geq d_{r, l} k^{\frac{(r-1) l}{l-1}},
$$


where

$$
d_{r, l}=\left[\frac{1}{\left(2^{r-1} r e\right)^{l}} \prod_{i=1}^{l-1}\left(1-\frac{i}{r}\right)\right]^{\frac{1}{l-1}}
$$

It is easy to see that this implies the result stated in the abstract. In the case where $r$ is fixed, we match the order of magnitude of the upper bound of Theorem 1.

Theorem 3 Let $r>l \geq 2$ be fixed. Then there exists $c$ depending only on $r$ and $l$ such that, for sufficiently large $k$ we have $m_{k}(r, l) \geq c\left(k^{r-1} \ln k\right)^{\frac{l}{l-1}}$.

We prove Theorem 1 in section 2 and Theorem 2 in section 3 . In section 4 we generalize a result from [13] about the chromatic number of hypergraphs with large independent sets; this result is used in section 5 in the proof of Theorem 3.

\section{The upper bound}

The bounds of the kind $(2)$ in $[13,15,11]$ hold for all $r$ and $k$, but apply only to large $k$ as written. Our construction also works for every $r>2$ and $k \geq 2$. It is an example of a random greedy algorithm.

Proof of Theorem 1: Consider the following procedure:

(1) order all $r$-element subsets of the set $\{1,2, \ldots, n\}$ at random: $R_{1}, \ldots, R_{\left(\begin{array}{c}n \\ r\end{array}\right)}$;

(2) Construct the family $G_{0}, \ldots, G_{\left(\begin{array}{c}n \\ r\end{array}\right)}$ of hypergraphs with the vertex set $V \stackrel{r}{=}\{1, \ldots, n\}$ as follows: $G_{0}$ has no edges and for $j=1, \ldots,\left(\begin{array}{l}n \\ r\end{array}\right)$ if $G_{j-1}+R_{j}$ is an $(r, l)$-system, then we let $G_{j}=G_{j-1}+R_{j}$, otherwise, $G_{j}=G_{j-1}$;

(3) Let $G(n, r)=G_{\left(\begin{array}{l}n \\ r\end{array}\right)}$.

Clearly, Part (2) is a deterministic procedure once the ordering is defined. Our aim is to prove that if $n=\left\lceil c_{r, l}\left(k^{r-1} \ln e k\right)^{\frac{1}{l-1}}\right\rceil$, where $c_{r, l}=\left(2 r^{3 l}\right)^{\frac{1}{l-1}}$ then with positive probability $G(n, r)$ has no independent set of size $\lceil n / k\rceil$. Thus such a hypergraph has no $k$-colorings. Since $G(n, r)$ is an $(r, l)$-system by construction, this will give us (for $r \geq 3$ ) an example of an $(r, l)$-system with chromatic number at least $k+1$ and the number of edges at most

$$
\frac{\left(\begin{array}{l}
n \\
l
\end{array}\right)}{\left(\begin{array}{l}
r \\
l
\end{array}\right)} \leq \frac{n(n-1)^{l-1}}{(r)_{l}} \leq 2 \frac{c_{r, l}^{l}}{(r)_{l}}\left(k^{r-1} \ln e k\right)^{\frac{l}{l-1}} .
$$

The proof follows from the following claim.

Claim. For an arbitrary set $X$ of vertices in $G(n, r)$ of cardinality $x=\lceil n / k\rceil$, the probability that $X$ induces no edges in $G(n, r)$ is less than $\left(\begin{array}{l}n \\ x\end{array}\right)^{-1}$. 
Proof. Fix an $X$ of size $x=\lceil n / k\rceil$. Let $B_{X}$ be the event that $X$ induces no edges in $G(n, r)$. Observe that $B_{X}$ implies that every $r$-set $T \subseteq X$ must be preceded (in the random ordering) by some $r$-set $R$ not in $X$ such that $R \in G(n, r)$ and $|R \cap T| \geq l$. Consequently, $l \leq|R \cap X| \leq r-1$. Let us call such an $R$ a witness for $T \in[X]^{r}$ not being included in $G(n, r)$. The point is that if $B_{X}$ happens, then we must have a large number of witnesses in $G(n, r)$, and the probability of the latter is small. Indeed, each $R \in G(n, r)$ can be a witness for at most $\left(\begin{array}{c}r-1 \\ l\end{array}\right)\left(\begin{array}{l}x-l \\ r-l\end{array}\right) r$-sets $T \subset X$. This means that in order to prevent all $\left(\begin{array}{l}x \\ r\end{array}\right) r$-sets $T$ of $X$ to appear in $G(n, r)$, the number of witnesses has to be at least

$$
m=\left\lceil\frac{\left(\begin{array}{l}
x \\
r
\end{array}\right)}{\left(\begin{array}{c}
r-1 \\
l
\end{array}\right)\left(\begin{array}{c}
x-l \\
r-l
\end{array}\right)}\right\rceil=\left\lceil\frac{(x)_{l}}{(r)_{l}\left(\begin{array}{c}
r-1 \\
l
\end{array}\right)}\right\rceil .
$$

For $j \geq 1$, let $A_{j}=A_{X, j}$ denote the event that the first $j$ edges $R_{l_{1}}, R_{l_{2}}, \ldots, R_{l_{j}}$ in $G(n, r)$ such that $\left|R_{l_{i}} \cap X\right| \geq l$ are not contained in $X$, i.e. $l \leq\left|R_{l_{i}} \cap X\right| \leq r-1$. The previous paragraph yields that if $B_{X}$ occurs, then $A_{m}$ also occurs.

The rest of the proof consists of bounding the probability of $A_{m}$ from above by $\left(\begin{array}{l}n \\ x\end{array}\right)^{-1}$.

For this calculation, we further assume that $R_{l_{1}}$ is the witness that appears first in the ordering, and that for each $1<j \leq m, R_{l_{j}}$ is the first witness which comes after $R_{l_{j-1}}$. Let $G^{j}=G_{l_{j}-1}$ be the family of all $r$-sets included in $G(n, r)$ before the $j$ th witness $R_{l_{j}}$ is chosen. For $1 \leq j \leq m$, let $\mathcal{S}_{j}$ be the collection of all $r$-sets $S$, such that $|X \cap S| \geq l$ and $|R \cap S|<l$ for all $R \in G^{j}$.

Since $A_{m} \subset A_{m-1} \subset \cdots \subset A_{2} \subset A_{1}$, we have

$$
\mathbf{P}\left\{A_{m}\right\}=\mathbf{P}\left\{A_{1}\right\} \cdot \mathbf{P}\left\{A_{2} \mid A_{1}\right\} \cdot \ldots \cdot \mathbf{P}\left\{A_{m} \mid A_{m-1}\right\}
$$

To estimate these probabilities we first note that each of the events $A_{1}$ and $A_{j+1} \mid A_{j}, j=$ $1, \ldots, m-1$ corresponds to a random choice from the set $\mathcal{S}_{j}$ with the result that the chosen set belongs to $\mathcal{S}_{j}-[X]^{r}$.

Since $\left|\mathcal{S}_{1}\right| \leq\left(\begin{array}{c}x \\ l\end{array}\right)\left(\begin{array}{c}n \\ r-l\end{array}\right)$ we have

$$
\mathbf{P}\left\{A_{1}\right\}=\frac{\left|\mathcal{S}_{1}\right|-\left(\begin{array}{l}
x \\
r
\end{array}\right)}{\left|\mathcal{S}_{1}\right|} \leq 1-\frac{\left(\begin{array}{l}
x \\
r
\end{array}\right)}{\left(\begin{array}{l}
x \\
l
\end{array}\right)\left(\begin{array}{c}
n \\
r-l
\end{array}\right)} .
$$

Furthermore, suppose that $j>1$, and let $j \leq m_{0}=\left\lceil\frac{m}{2}\right\rceil$. Assume now that the event $A_{j}$ occurred. Since

$$
\mathbf{P}\left\{A_{j} \mid A_{j-1}\right\}=\frac{\left|\mathcal{S}_{j}-[X]^{r}\right|}{\left|\mathcal{S}_{j}\right|}=1-\frac{\left|\mathcal{S}_{j} \cap[X]^{r}\right|}{\left|\mathcal{S}_{j}\right|},
$$

we need to estimate the cardinality of the set $\mathcal{S}_{j} \cap[X]^{r}$. 
The hypergraph $G^{j}$ contains precisely $j-1 r$-sets $R$ with $l \leq|R \cap X| \leq r-1$. Each of these is a witness for at most

$$
\left(\begin{array}{c}
|X \cap R| \\
l
\end{array}\right)\left(\begin{array}{l}
x-l \\
r-l
\end{array}\right) \leq\left(\begin{array}{c}
r-1 \\
l
\end{array}\right)\left(\begin{array}{l}
x-l \\
r-l
\end{array}\right)
$$

$r$-sets. Consequently, the number of $r$-sets $T$ in $X$ with no witness at this stage is

$$
\left|\mathcal{S}_{j} \cap[X]^{r}\right| \geq\left(\begin{array}{l}
x \\
r
\end{array}\right)-(j-1)\left(\begin{array}{c}
r-1 \\
l
\end{array}\right)\left(\begin{array}{l}
x-l \\
r-l
\end{array}\right) \geq\left(\begin{array}{l}
x \\
r
\end{array}\right)-\left(m_{0}-1\right)\left(\begin{array}{c}
r-1 \\
l
\end{array}\right)\left(\begin{array}{l}
x-l \\
r-l
\end{array}\right) \geq \frac{1}{2}\left(\begin{array}{l}
x \\
r
\end{array}\right),
$$

where the last inequality follows from the choice of $m_{0}$. Summarizing, we infer that

$$
\mathbf{P}\left\{A_{j} \mid A_{j-1}\right\} \leq 1-\frac{\frac{1}{2}\left(\begin{array}{l}
x \\
r
\end{array}\right)}{\left(\begin{array}{c}
n \\
r-l
\end{array}\right)\left(\begin{array}{l}
x \\
l
\end{array}\right)} .
$$

This yields

$$
\begin{gathered}
\mathbf{P}\left\{A_{m}\right\} \leq \mathbf{P}\left\{A_{m_{0}}\right\}=\mathbf{P}\left\{A_{1}\right\} \cdot \mathbf{P}\left\{A_{2} \mid A_{1}\right\} \cdot \ldots \cdot \mathbf{P}\left\{A_{m_{0}} \mid A_{m_{0}-1}\right\} \leq \\
\leq\left(1-\frac{\frac{1}{2}\left(\begin{array}{c}
x \\
r
\end{array}\right)}{\left(\begin{array}{c}
n \\
r-l
\end{array}\right)\left(\begin{array}{l}
x \\
l
\end{array}\right)}\right)^{m_{0}} \leq\left(1-\frac{\frac{1}{2}\left(\begin{array}{c}
x \\
r
\end{array}\right)}{\left(\begin{array}{c}
n \\
r-l
\end{array}\right)\left(\begin{array}{c}
x \\
l
\end{array}\right)}\right)^{\frac{(x)_{l}}{2(r) l\left(\begin{array}{c}
r-1 \\
l
\end{array}\right)}} \leq \exp \left\{-\frac{(x)_{r}}{4 n^{r-l(}\left(\begin{array}{c}
r \\
l
\end{array}\right)(r)_{l}\left(\begin{array}{c}
r-1 \\
l
\end{array}\right)}\right\} .
\end{gathered}
$$

In order to prove the claim we will show that the last expression is less than $\left(\begin{array}{l}n \\ x\end{array}\right)^{-1}$. Since $\left(\begin{array}{l}n \\ x\end{array}\right)<(n e / x)^{x}=\exp \left\{x \ln \frac{e n}{x}\right\}$, and $x=\lceil n / k\rceil$, we have

$$
\mathbf{P}\left\{A_{m}\right\} \cdot\left(\begin{array}{l}
n \\
x
\end{array}\right)<\exp \left\{x\left(\ln \frac{e n}{x}-\frac{(x-1)_{r-1}}{4 n^{r-l}\left(\begin{array}{l}
r \\
l
\end{array}\right)(r)_{l}\left(\begin{array}{c}
r-1 \\
l
\end{array}\right)}\right)\right\} .
$$

By the choice of $n$, it is easy to observe that $x \geq n / k \geq(r-1)^{3}$. Thus for $r \geq 3$

$$
(x-1)_{r-1} \geq\left(1-\frac{r-1}{x}\right)^{r-1} x^{r-1} \geq\left(1-\frac{1}{(r-1)^{2}}\right)^{r-1} x^{r-1} \geq \frac{1}{2} x^{r-1} \geq \frac{1}{2}\left(\frac{n}{k}\right)^{r-1} .
$$

Consequently, $\mathbf{P}\left\{A_{m}\right\} \cdot\left(\begin{array}{l}n \\ x\end{array}\right)$ is strictly less than

$$
\exp \left\{x\left(\ln e k-\frac{\frac{1}{2}\left(\frac{n}{k}\right)^{r-1}}{4 n^{r-l}\left(\begin{array}{c}
r \\
l
\end{array}\right)(r)_{l}\left(\begin{array}{c}
r-1 \\
l
\end{array}\right)}\right)\right\} \leq 1,
$$

where the last inequality follows since

$$
n \geq\left(2 r^{3 l}\right)^{\frac{1}{l-1}}\left(k^{r-1} \ln e k\right)^{\frac{1}{l-1}} \geq\left[8\left(\begin{array}{c}
r \\
l
\end{array}\right)(r)_{l}\left(\begin{array}{c}
r-1 \\
l
\end{array}\right)\right]^{\frac{1}{l-1}}\left(k^{r-1} \ln e k\right)^{\frac{1}{l-1}} .
$$




\section{Lower bounds from the Lovász Local Lemma}

In this section, we prove Theorem 2. Our main tool is the symmetric version of the Lovász Local Lemma which we state below (see [5] for a proof).

Lemma 4 (Local Lemma) Let $A_{1}, \ldots, A_{n}$ be events in a probability space. Suppose that each event $A_{i}$ is mutually independent of a set of all the other events $A_{j}$ but at most $d$, and that $\operatorname{Prob}\left[A_{i}\right] \leq p$ for all $i$. If ep $(d+1) \leq 1$, then $\operatorname{Prob}\left[\wedge \overline{A_{i}}\right]>0$.

We use the following lemma from [10], whose proof we supply for completeness.

Lemma 5 Let $H$ be an $r$-graph. If every vertex in $H$ has degree at most $k^{r-1} /$ er, then $\chi(H) \leq k$.

Proof. We color the vertices of $H$ with $k$ colors, with each color being assigned to each vertex independently with equal probability. The probability that a given edge is monochromatic is $1 / k^{r-1}$. The event $A_{F}$ that edge $F$ is monochromatic is independent of all events $A_{F^{\prime}}$ with $F \cap F^{\prime}=\emptyset$. The number of $F^{\prime}$ with $F \cap F^{\prime} \neq \emptyset$ is at most $r\left(k^{r-1} /(e r)-1\right) \leq k^{r-1} / e-1$. The Local Lemma (Lemma 4 ) therefore implies that there is a $k$-coloring with no monochromatic edge.

Proof of Theorem 2: Let $H$ be an $(r, l)$-system with at most $z=c_{r, l} k^{\frac{(r-1) l}{l-1}}$ edges, where

$$
c_{r, l}=\left[\frac{1}{\left(2^{r-1} r e\right)^{l}} \prod_{i=1}^{l-1}\left(1-\frac{i}{r}\right)\right]^{\frac{1}{l-1}} .
$$

Let

$$
A=\left\{v \in V(H): \operatorname{deg}(v)>\frac{k^{r-1}}{e r 2^{r-1}}\right\} .
$$

Let $B=V(H)-A$, and let $H_{A}$ and $H_{B}$ be the subhypergraphs induced by $A$ and $B$, respectively. By Lemma 5 , there is a proper $k / 2$-coloring of $H_{B}$.

Now color $H_{A}$ randomly using a new set of $k / 2$ colors, where each color appears on each vertex independently with equal probability. Since $H$ is an $(r, l)$-system, every vertex in $H_{A}$ has degree (in $H_{A}$ ) at most

$$
\Delta=\frac{(a-1)(a-2) \cdots(a-l+1)}{(r-1)(r-2) \cdots(r-l+1)}
$$


where $a=|A|$. Consequently, each edge $E$ in $H_{A}$ is incident with at most $d=\Delta r-r$ other edges. Moreover, since $H$ has at most $z$ edges and $z r>a k^{r-1} /\left(e r 2^{r-1}\right)$, we infer that $a<z e r^{2} 2^{r-1} / k^{r-1}$. Consider the space of colorings with each vertex being colored randomly and independently of others. For each edge $E$ in $H_{A}$, let $M_{E}$ be the event that $E$ is monochromatic. Since $p=\mathbf{P}\left\{M_{E}\right\}=(2 / k)^{r-1}$ and

$$
\begin{aligned}
e p(d+1) & \leq e\left(\frac{2}{k}\right)^{r-1}\left(\frac{(a-1)(a-2) \cdots(a-l+1)}{(r-1)(r-2) \cdots(r-l+1)} r-r+1\right) \leq \\
& \leq e\left(\frac{2}{k}\right)^{r-1} \frac{a^{l-1} r}{(r-1)(r-2) \cdots(r-l+1)} \leq \\
& \leq e^{l} \frac{r^{2 l-1}}{(r-1) \cdots(r-l+1)}\left(\frac{2}{k}\right)^{(r-1) l} z^{l-1}<1,
\end{aligned}
$$

the Local Lemma implies that there is a proper $k / 2$-coloring of $H_{A}$. These two colorings together yield a proper $k$-coloring of $H$.

\section{From independent sets to proper colorings}

In this section, we prove a preliminary Lemma 8 to our main lower bound, Theorem 3, which might be interesting of its own. A special case appears in [13]. The following fact was kindly pointed out to us by a referee.

Lemma 6 Let $f(m)$ be a monotonically non-decreasing function, $f(1)=1$, and $f(m) \leq m$ for every $m$. Let $G$ be a graph on $n$ vertices. Let $I_{1}, \ldots, I_{t}$ be a family of disjoint independent sets in $G$ with $i_{l}=\left|I_{l}\right|$ for $l=1, \ldots, t$. Let $x_{0}=0$ and $x_{l}=\sum_{j=1}^{l} i_{j}$. If $i_{j} \geq f\left(n-x_{j-1}\right)$ for every $j=1, \ldots, t$, then $t \leq \sum_{l=n-x_{t}+1}^{n} \frac{1}{f(l)}$.

Proof. Since $f(m)$ is monotonically non-decreasing and $i_{j} \geq f\left(n-x_{j-1}\right)$, we have

$$
\sum_{l=n-x_{t}+1}^{n} \frac{1}{f(l)}=\sum_{j=1}^{t} \sum_{l=n-x_{j}+1}^{n-x_{j-1}} \frac{1}{f(l)} \geq \sum_{j=1}^{t} \sum_{l=n-x_{j}+1}^{n-x_{j-1}} \frac{1}{f\left(n-x_{j-1}\right)}=\sum_{j=1}^{t} \frac{i_{j}}{f\left(n-x_{j-1}\right)} \geq t .
$$

This lemma (due to a referee) directly implies the following nice corollary.

Lemma 7 Let $f(m)$ be a monotonically non-decreasing function, $f(1)=1$, and $f(m) \leq m$ for every $m$. Let $G$ be a graph on $n$ vertices. If for every $2 \leq m \leq n$, the independence number of every $m$-vertex subgraph of $G$ is at least $f(m)$, then $\chi(G) \leq \sum_{j=1}^{n} \frac{1}{f(j)}$. 
Lemma 8 Let $0 \leq \alpha<1$ and $\beta<1-\alpha$. Let $H$ be a hypergraph with $n$ vertices. Suppose that every subhypergraph $P$ of $H$ (including $H$ itself) with $m \geq 2$ vertices has an independent set of size at least $f(m)=\mathrm{cm}^{\alpha}(\ln e m)^{\beta}$ for some constant $c>0$. Then there is a $d=$ $d(c, \alpha, \beta)>0$ such that $\chi(H) \leq d n^{1-\alpha}(\ln e n)^{-\beta}$.

Proof. Define $f(1)=1$. Then by Lemma 7 ,

$$
\begin{gathered}
\chi(H) \leq \sum_{j=1}^{n} \frac{1}{f(j)} \leq 1+\int_{1}^{n} \frac{1}{c} x^{-\alpha}(\ln e x)^{-\beta} d x \leq \\
\leq 1+\frac{1}{c(1-\alpha-\beta)} \int_{1}^{n} x^{-\alpha}(\ln e x)^{-\beta}\left(1-\alpha-\frac{\beta}{\ln e x}\right) d x= \\
=1+\left.\frac{1}{c(1-\alpha-\beta)}\right|_{1} ^{n} x^{1-\alpha}(\ln e x)^{-\beta}=1+\frac{1}{c(1-\alpha-\beta)}\left(n^{1-\alpha}(\ln e n)^{-\beta}-1\right) .
\end{gathered}
$$

This proves the lemma.

We use Lemma 8 to prove that $(r, l)$-systems with not too many vertices are $k$-colorable. The following result of Rödl and Sinajová guarantees large independent sets in such $r$-graphs.

Theorem 9 (Rödl-S̆inajová [15]) Let $H$ be an $(r, l)$-system on $n$ vertices. Then $H$ has an independent set of size at least $c n^{(r-l) /(r-1)}(\ln n)^{1 /(r-1)}$, where $c$ is a positive constant depending only on $r$ and $l$.

Theorem 9 together with Lemma 8 implies

Theorem 10 Let $H$ be an $(r, l)$-system on $n$ vertices. Then $\chi(H) \leq c\left(n^{l-1} / \ln n\right)^{1 /(r-1)}$ for some constant $c$ depending only on $r$ and $l$. Moreover, there is another constant $c^{\prime}$ (also depending only on $r$ and $l$ ) such that, if $n \leq c^{\prime}\left(k^{r-1} \ln k\right)^{1 /(l-1)}$, then $\chi(H) \leq k$.

\section{The main lower bound}

In this section we prove Theorem 3 . The main idea to properly $k$-color the $(r, l)$-system is to greedily take maximal independent sets. We therefore need a lower bound on the size of a maximal independent set in an $r$-graph. Such a bound is provided for a fairly restricted class by a result of Ajtai et al. [3]. 
Theorem 11 ([3]) Let $G$ be an r-uniform hypergraph without 2-,3- and 4-cycles. If $|E(G)| /|V(G)|$ is very large in comparison with $r$, then

$$
\alpha(G) \geq c \frac{|V(G)|^{r /(r-1)}}{|E(G)|^{1 /(r-1)}}\left(\ln \frac{|E(G)|}{|V(G)|}\right)^{1 /(r-1)},
$$

where $c$ depends only on $r$.

We remark that the condition $|E(G)| /|V(G)|$ being large can be removed by changing the constant $c$. Duke et al. [7] extended this bound (with a different constant) to the class of simple hypergraphs. We need the following generalization of [7] for $(r, l)$-systems; the proof follows from the idea in [15].

Theorem 12 Let $r, l$ be integers with $r>l>1$, and let $\delta=(r-l) /(8 r-10)$. Suppose that $F$ is an $(r, l)$-system with $|V(F)|=n$ and $|E(F)| \geq n^{l-\delta}$. Then

$$
\alpha(F) \geq c_{1} n\left(\frac{\ln w}{w}\right)^{1 /(r-1)},
$$

where $w=|E(F)| / n$ and $c_{1}$ depends only on $r$ and $l$.

Proof. (Sketch) Let $\epsilon_{0}=(4 l-5) /(4 r-5)$ and $\epsilon_{1}=(l-1-\delta) /(r-1)<(l-1) /(r-1)$. Set $\epsilon=\left(\epsilon_{0}+\epsilon_{1}\right) / 2$. Consider a random induced subsystem $H$ of $F$, where every vertex in $H$ is included with probability $p=n^{-\epsilon}$ independently of all other vertices. The expected number of vertices in $H$ is $p n$ and the expected number of edges in $H$ is $p^{r}|E(F)|$. In [15] it is proven that with positive probability, we can delete at most half of the vertices of $H$ to obtain a subsystem $G$ of $F$ with

(1) no cycles of length less than five,

(2) $\mid V(G)=p n / 2$, and

(3) $|E(G)| \leq 2 p^{r}|E(F)|$.

We apply Theorem 11 to $G$. With given $|V(G)|$, the bound we seek for $\alpha(G)$ decreases when $|E(G)|$ grows. Therefore, letting $z=|E(F)|$, we obtain that $\alpha(F)$ is at least

$$
\alpha(G) \geq c \frac{(p n / 2)^{\frac{r}{r-1}}}{\left(2 p^{r} z\right)^{\frac{1}{r-1}}}\left(\ln \frac{4 p^{r} z}{p n}\right)^{\frac{1}{r-1}}=c \frac{(n / 2)^{\frac{r}{r-1}}}{(2 z)^{\frac{1}{r-1}}}\left(\ln \frac{4 z}{n^{\epsilon(r-1)+1}}\right)^{\frac{1}{r-1}} \geq c_{1} \frac{n^{\frac{r}{r-1}}}{z^{\frac{1}{r-1}}}\left(\ln \frac{z}{n}\right)^{\frac{1}{r-1}} .
$$

The last inequality follows by replacing the exponent inside the logarithm by a factor outside of the logarithm. 
Proof of Theorem 3: Let $H$ be an $(r, l)$-system with at most $c_{2}\left(k^{r-1} \ln k\right)^{\frac{l}{l-1}}$ edges. Set $c_{3}=1 /\left(e r 3^{r-1}\right)$. Partition $V(H)$ into two parts:

$V_{0}$ - vertices of degree at most $c_{3} k^{r-1}$, and

$V_{1}$ - vertices of degree greater than $c_{3} k^{r-1}$.

By Lemma 5 there is a proper coloring of the vertices in $V_{0}$ with $k / 3$ colors. It remains to properly color the vertices of $V_{1}$ with at most $2 k / 3$ colors.

Let $H_{1}=H\left(V_{1}\right)$ and $n_{1}=\left|V_{1}\right|$. Let $c_{4}$ be chosen so that by Theorem 10, every $(r, l)$ system with at most

$$
n_{0}=c_{4}\left(k^{r-1} \ln k\right)^{1 /(l-1)}
$$

vertices is $\frac{k}{3}$-colorable. Because

$$
n_{1} c_{3} k^{r-1} \leq \sum_{v \in V_{1}} \operatorname{deg}(v) \leq r|E(H)|
$$

we obtain

$$
n_{1} \leq \frac{r c_{2}}{c_{3}}\left(k^{r-1} \ln ^{l} k\right)^{1 /(l-1)}=\frac{r c_{2}}{c_{3} c_{4}} n_{0} \ln k .
$$

Let $a_{1}=\frac{n_{1}}{n_{0}}$. If $a_{1} \leq 1$, then we are done, and by (6), in any case,

$$
a_{1} \leq \frac{r c_{2}}{c_{3} c_{4}} \ln k
$$

Let $i \geq 1$ and consider the following procedure:

\section{Step $i$ :}

(a) If $a_{i}>1$, then we distinguish between two cases depending on whether $\left|E\left(H_{i}\right)\right|$ is large.

(I) If $\left|E\left(H_{i}\right)\right| \geq n_{i}^{l-\delta}$, where $\delta$ is as in Theorem 12, then we can apply Theorem 12 . Choose in $H_{i}$ a maximum independent set $I_{i}$, let $H_{i+1}=H_{i}-I_{i}, n_{i+1}=\left|V\left(H_{i+1}\right)\right|=n_{i}-\left|I_{i}\right|$ and $a_{i+1}=\frac{n_{i+1}}{n_{0}}$. Now go to Step $i+1$.

(II) If $\left|E\left(H_{i}\right)\right|<n_{i}^{l-\delta}$, then partition $V\left(H_{i}\right)$ into two sets $X$ and $Y$, where $X$ consists of all vertices of $H_{i}$ with degree less than $d k^{r-1}$, with $d=1 /\left(\operatorname{er} 6^{r-1}\right)$.

By the choice of $d$, Lemma 5 implies that the hypergraph induced by $X$ can be properly $k / 6$-colored. Let $d^{\prime}$ be chosen so that, by Theorem 10 , every $(r, l)$-system on at most $d^{\prime}\left(k^{r-1} \ln k\right)^{1 /(l-1)}$ vertices is properly $k / 6$-colorable. Because

$$
|Y| d k^{r-1} \leq \sum_{v \in Y} \operatorname{deg}(v) \leq r\left|E\left(H_{i}\right)\right| \leq r n_{i}^{l-\delta}<r n_{1}^{l-\delta}
$$

we conclude that since $k$ is sufficiently large

$$
|Y| \leq \frac{r n_{1}^{l-\delta}}{d k^{r-1}} \leq \frac{r}{d k^{r-1}}\left[\frac{r c_{2}}{c_{3}} \ln k\left(k^{r-1} \ln k\right)^{\frac{1}{l-1}}\right]^{l-\delta} \leq d^{\prime}\left(k^{r-1} \ln k\right)^{\frac{1}{l-1}}
$$


Consequently, the subhypergraph of $H_{i}$ induced by $Y$ can be properly $k / 6$-colored. These two colorings together yield a proper $k / 3$-coloring of $H_{i}$. Color $H_{i}$ properly with $k / 3$ colors. Since all vertices of $H$ are now colored, we stop the procedure.

(b) if $a_{i} \leq 1$, then the number of vertices in the uncolored hypergraph is at most $n_{0}$. We apply Theorem 10 to color these vertices with $k / 3$ colors. Now we stop the procedure.

Suppose that the procedure stops on Step $t+1$. That means that in Steps $i=1, \ldots, t$, we were in Case (a) part (I). We will prove that $t \leq k / 3$. Observe that this implies that $H$ is $k$-colorable.

$(\alpha)$ We used $k / 3$ colors to color $H\left(V_{0}\right)$,

( $\beta)$ We used $t$ colors for $I_{1}, \ldots, I_{t}$,

$(\gamma)$ Regardless of whether we stopped the procedure due to Case (a) part (II), or Case (b), in each situation we used $k / 3$ new colors. This yields the required $k$-coloring of $H$.

In order to complete the argument, we will show that in $(\beta)$ we have $t \leq k / 3$.

By the definition of $a_{i}$, we have

$$
\left|E\left(H_{i}\right)\right| /\left|V\left(H_{i}\right)\right| \leq \frac{c_{2}\left(k^{r-1} \ln k\right)^{l /(l-1)}}{a_{i} c_{4}\left(k^{r-1} \ln k\right)^{1 /(l-1)}} \leq \frac{c_{2}\left(k^{r-1} \ln k\right)^{l /(l-1)}}{a_{i} c_{4}\left(k^{r-1} \ln k\right)^{1 /(l-1)}}=\frac{c_{2} k^{r-1} \ln k}{a_{i} c_{4}}
$$

and hence by Theorem 12 for large $k$,

$$
\left|I_{i}\right| \geq c_{1} n_{i}\left(\frac{a_{i} c_{4}}{c_{2} k^{r-1} \ln k}\right)^{\frac{1}{r-1}}\left(\ln \frac{c_{2} k^{r-1} \ln k}{a_{i} c_{4}}\right)^{\frac{1}{r-1}} \geq\left(\frac{a_{i} c_{4}}{c_{2}}\right)^{\frac{1}{r-1}} \frac{c_{1} n_{i}}{k}=\left(\frac{c_{4}}{c_{2} n_{0}}\right)^{\frac{1}{r-1}} \frac{c_{1} n_{i}^{\frac{r}{r-1}}}{k} .
$$

Let $c_{5}=c_{1}\left(\frac{c_{4}}{c_{2}}\right)^{\frac{1}{r-1}}$. Then by (9), the conditions of Lemma 6 are satisfied with $f(m)=$ $\frac{c_{5} n_{i}^{\frac{r}{r-1}}}{k n_{0}^{\frac{1}{r-1}}}$. Hence by Lemma 6 ,

$$
\begin{gathered}
t \leq 1+\sum_{l=n_{0}+1}^{n} \frac{1}{f(l)}=1+\frac{k n_{0}^{\frac{1}{r-1}}}{c_{5}} \sum_{l=n_{0}+1}^{n} l^{-\frac{r}{r-1}} \leq 1+\frac{k n_{0}^{\frac{1}{r-1}}}{c_{5}} \int_{n_{0}}^{n} x^{-\frac{r}{r-1}} d x \leq \\
\leq 1+\frac{k(r-1) n_{0}^{\frac{1}{r-1}}}{c_{5}}\left(n_{0}^{\frac{-1}{r-1}}-n^{\frac{-1}{r-1}}\right) \leq 1+\frac{k(r-1)}{c_{5}} .
\end{gathered}
$$

Thus if we choose $c_{2}$ small enough to make $c_{5}>6(r-1)$, then for large $k$ we will have $t<k / 3$. This proves the bound.

Acknowledgment. We sincerely thank a referee for Lemma 6 simplifying our previous calculations and for the other helpful suggestions. 


\section{References}

[1] N. Alon, Hypergraphs with high chromatic number, Graphs and Combinatorics, 1, 387389, 1985.

[2] H. Abbot, D. Hanson, On a combinatorial problem of Erdős, Canad. Math. Bull. 12, 823-829, 1969.

[3] M. Ajtai, J. Komlós, J. Pintz, J. Spencer, E. Szemerédi, Extremal uncrowded hypergraphs, J. Combinatorial Theory, Ser. A, 32, 321-335, 1982.

[4] P. Aizley, J. Selfridge. Abstract. Notices Amer. Math. Soc. 24, A-452, 1977.

[5] N. Alon, J. H. Spencer, The Probabilistic Method, Wiley-Interscience Series, John Wiley \& Sons, Inc., New York, 1992.

[6] J. Beck, On 3-chromatic hypergraphs, Discrete Mathematics, 24, 127-137, 1978.

[7] R. Duke, H. Lefmann, V. Rödl, On uncrowded hypergraphs, Random Structures and Algorithms, 6, 209-212, 1995.

[8] P. Erdős, On a combinatorial problem, I, Nordisk Mat. Tidskrift, 11, 5-10, 1963.

[9] P. Erdős, On a combinatorial problem, II, Acta Mathematica of the Academy of Sciences, Hungary, 15, 445-447, 1964.

[10] P. Erdős, L. Lovász, Problems and results on 3-chromatic hypergraphs and some related questions, In Infinite and Finite Sets, A. Hajnal et al., editors, Colloq. Math. Soc. J. Bolyai 11, North Holland, Amsterdam, 609-627, 1975.

[11] D. Grable, K. Phelps, V. Rödl, The minimum independence number for designs, Combinatorica 15, 175-185, 1995.

[12] T. Jensen, B. Toft, Graph Coloring Problems, Wiley Interscience Series, John Wiley \& Sons, Inc., p. 231, 1995.

[13] K. Phelps, V. Rödl, Steiner triple systems with minimum independence number, Ars Combinatoria 21, 167-172, 1986.

[14] J. Radhakrishnan, A. Srinivasan, Improved bounds and algorithms for hypergraph twocoloring, Random Structures and Algorithms, 16, 4-32, 2000. 
[15] V. Rödl, E. Šinajová, Note on independent sets in Steiner systems, Random Structures and Algorithms, 5, 183-190, 1994. 